\title{
Pulmonary complications and respiratory function changes after bone marrow transplantation in children
}

\author{
F. Fanfulla*, F. Locatelli**, M.C. Zoia*, G. Giorgiani**, \\ F. Bonetti**, L. Spagnolatti*, I. Cerveri*
}

Pulmonary complications and respiratory function changes after bone marrow transplantation in children. F. Fanfulla, F. Locatelli, M.C. Zoia, G. Giorgiani, F. Bonetti, L. Spagnolatti, I. Cerveri. @ ERS Journals Ltd 1997.

ABSTRACT: We prospectively assessed the frequency of pulmonary complications and the natural course of lung function after bone marrow transplantation (BMT), as well as the effect of several risk factors in a homogeneous group of 39 children who underwent allogeneic or autologous BMT for haematological malignancies between 1992 and 1995.

Four patients developed pneumonia within the first 3 months and three 3-6 months after BMT. A considerable percentage of acute bronchitis was recorded throughout the follow-up. Three patients died after the 6 month visit because of pneumonia (two patients) and pulmonary aspergillosis (one patient). No patients had obstructive lung disease syndrome. At 3 months after BMT, forced vital capacity (FVC), forced expiratory volume in one second (FEV1) and transfer factor of the lung for carbon monoxide ( $T \mathrm{~L}, \mathrm{CO}$ ) significantly decreased, but $\mathrm{FEV} 1 / \mathrm{FVC}$ ratio and maximal expiratory flow at $25 \%$ of $\mathrm{FVC}$ remained unchanged, suggesting a restrictive defect with diffusion impairment. At 18 months, there was a progressive recovery in lung function, although only 11 patients had normalized. Seropositivity for cytomegalovirus had a significant effect on lung function whereas graft-versus host disease also had an effect, although it was not statistically significant. Baseline respiratory function, type of transplant, type of conditioning regimen and respiratory infections did not significantly affect the outcome of BMT.

The high frequency of severe lung function abnormalities found in this study, suggests a careful functional monitoring in all subjects undergoing bone marrow transplantation, even in the absence of respiratory symptoms. Eur Respir J 1997; 10: 2301-2306.

The increasing success of bone marrow transplantation (BMT) for treatment of haematological malignancies and solid tumours has now drawn much attention to the late sequelae of therapy on the function of several systems.

Pulmonary complications are a major cause of morbidity and mortality in adult BMT recipients $[1,2]$. There is little published information on pulmonary complications and respiratory function changes occurring in children who received BMT [3]. Most previous studies reported only retrospective analyses of serial pulmonary function tests in children who received BMT $[4,5]$, and longitudinal pulmonary function studies in this population are sparse $[6,7]$. Furthermore, available data are conflicting to some extent [4-6, 8]. Variance between studies may result from the different underlying diseases and the changes of therapeutic strategies over time.

Recent advances in transplant immunobiology, supportive care and prevention of graft-versus-host disease (GVHD) have markedly changed the outcome of BMT. We undertook this prospective study to verify the frequency of pulmonary complications, the natural course of lung function after BMT and the effect on it of several risk factors in a homogeneous group of children
*Institute of Respiratory Diseases, and **Dept of Paediatrics, IRCCS Policlinico "S.Matteo", University of Pavia, Italy.

Correspondence: I. Cerveri

Istituto di Tisiologia e Malattie dell'Apparato Respiratorio

V. Taramelli 5

27100 Pavia

Italy

Keywords: Bone marrow transplantation children

lung function

pulmonary complications

Received: December 131996

Accepted after revision June 221997 who underwent BMT over a short period, when therapeutic strategies remained essentially unchanged.

\section{Patients and methods}

\section{Study protocol and patient selection}

Thirty nine children (22 males, 17 females) aged $\geq 6$ yrs, who received an autologous (10 patients) or allogeneic (29 patients) BMT between September 1992 and September 1995, were enrolled in this follow-up study. Written informed consent was obtained from their parents. Clinical examination, chest radiograph and lung function tests were obtained before BMT. Follow-up visits were scheduled for 3, 6, 12 and 18 months afterwards. Respiratory symptoms present at the follow-up visit were recorded, as well as any significant respiratory problems occurring between visits.

The total number of patients undergoing BMT in the period considered was 56 , but 17 children were excluded because they were unable to perform pulmonary function tests (three patients), or showed an early relapse of disease (five patients), or died of transplantrelated causes in the first 3 months after BMT (nine patients, two of whom experienced idiopathic pneumonia 
syndrome). The 39 patients included had a minimum follow-up of 3 months. At 6 months after BMT, two patients had been lost to follow-up: one had died of relapse and one had developed encephalitis. At 12 months after BMT, six other patients were no longer evaluable: five had relapsed (four died) and one had died of pneumonia causing respiratory failure. At 18 months after BMT, three other patients had died (one of relapse, one of pulmonary aspergillosis with respiratory failure, one of fungal meningitis) and one did not attend the hospital for follow-up visit because of family problems. The characteristics of the patients included in the study are reported in table 1 . Twenty children had been transplanted for acute lymphoblastic leukaemia (ALL), 13 for acute myelogenous leukaemia (AML), three for chronic myelogenous leukaemia (CML), and three for nonHodgkin's lymphoma (NHL). Before BMT, patients with ALL, AML and NHL had been previously treated using protocols established by the Italian Association for Pediatric Hematology/Oncology, whereas those with CML had received hydroxyurea $[9,10]$. In particular, the majority of patients with ALL were given BMT after a second haematological remission, having experienced a medullary relapse, whereas children with AML were mainly transplanted at the first haematological remission. The median interval between diagnosis and BMT for children with ALL and AML was 30 and 5 months, respectively.

Twenty nine patients were given a conditioning regimen including fractionated total body irradiation (TBI; 12 Gy divided into six fractions over 3 days) associated with cytotoxic drugs, such as cyclophosphamide (CY), thiotepa (TT), melphalan (L-PAM), carmustine (BCNU) and vincristine (VCR). Eight children underwent BMT after a myeloablative therapy consisting of busulfan (BU) (16 mg. $\mathrm{kg}^{-1}$ p.o. over 4 days) and CY (120 mg. $\mathrm{kg}^{-1}$ i.v.), with (six patients) or without (two patients) L-PAM (140 $\mathrm{mg} \cdot \mathrm{m}^{-2}$.single dose $\mathrm{e}^{-1}$ i.v.). The remaining two children underwent BMT after a regimen of etoposide/TT/ $\mathrm{CY}$ and BCNU/TT/L-PAM, respectively. GVHD prophylaxis consisted of cyclosporin-A (Cs-A) in 20 children and methotrexate (MTX) in two children given an

Table 1. - Characteristics of the 39 patients enrolled in the study

\begin{tabular}{lr}
\hline Age yrs & 11 (6-18) \\
Sex M/F & $22 / 17$ \\
Type of BMT n & 29 \\
$\quad$ Allogeneic & 10 \\
$\quad$ Autologous & \\
HLA compatibility of donors n & 22 \\
$\quad$ Matched siblings & 7 \\
$\quad$ Matched unrelated donors & $12(1-42)$ \\
Age at BMT yrs & \\
Conditioning regimens n & 29 \\
$\quad$ TBI/cytoxic drugs & 6 \\
BU/CY/L-PAM & 2 \\
BU/CY & 2 \\
Other regimens & 24 \\
HCMV-seropositive donor and/or &
\end{tabular}

Values are presented as absolute numbers, or as medians, with ranges in parentheses. M: male; F: female; HLA: human major histocompatability complex; BMT: bone marrow transplantation; TBI: total body irradiation; BU: busulfan; CY: cyclophosphamide; L-PAM: melphalan; HCMV: human cytomegalovirus. For further details see also Patients and methods section. allogeneic BMT from an HLA-identical sibling, while seven patients receiving a matched unrelated transplant were treated, in addition to Cs-A, with short-course MTX [11] and the monoclonal antibody Campath-1G in vivo.

Acute GVHD occurrence was classified according to previously described criteria [12] and treated with steroids as first-line therapy and horse antilymphocyte globulin in resistant cases. Chronic GVHD was diagnosed according to the criteria published by SHULmAN et al. [13] and treated using Cs-A and steroids when necessary. All 12 children who developed grade II-IV acute GVHD recovered after the treatment mentioned above, while 10 of the patients with acute GVHD developed limited chronic GVHD, which required therapy in four cases. Twenty of the 29 patients given allogeneic BMT had positive serology for human cytomegalovirus (HCMV), whereas 21 donors were seropositive.

\section{Respiratory symptoms and complications}

At each scheduled visit, the presence of cough and/or phlegm, dyspnoea and wheezing, not sufficiently indicative of a specific pathology, was recorded and coded. When acute bronchitis or pneumonia were diagnosed at the scheduled time of the visit, the respiratory function tests had to be delayed until at least 10 days after recovery. Acute bronchitis was defined as a clinically apparent acute episode of airway inflammation with cough and mucopurulent phlegm requiring treatment; pneumonia was defined as the occurrence of an acute pulmonary infection, with physical and radiographic signs of parenchymal thickening. Isolation of bacterial, mycotic and viral agents from secretions obtained by deep coughing was attempted. Children with pneumonia were treated using broad spectrum antibiotic therapy (i.e. aminoglycosides and second generation cephalosporine). If fever persisted for $48 \mathrm{~h}$, vancomycin was added, whereas antifungal agents were employed if fever persisted for a further $24 \mathrm{~h}$. If sputum cultures demonstrated a specific organism, appropriate antibiotic therapy was administered. Antibiotic or antifungal treatment was discontinued after complete resolution of the infections and normalization of the chest radiograph.

The diagnosis of obstructive lung disease was made when patients met all the following criteria: 1) no evidence of pulmonary abnormalities before transplantation; 2) cough, wheezing, and/or dyspnoea in the absence of infection; 3) forced expiratory volume in one second (FEV1) and maximal expiratory flow at $25 \%$ of forced vital capacity (MEF25) SD score <-1.64. All the respiratory diagnoses were made by the same team of clinicians and all chest radiographs reviewed by one of the investigators.

\section{Pulmonary function tests}

Measurements of lung volumes were obtained by a water-sealed spirometer (Pulmonet III, Sensor Medics, Anaheim, CA, USA). Measurements were performed according to the European Coal and Steel Community (ECSC) statements [14] and to the American Thoracic Society (ATS) recommendations [15]. The best of three forced vital capacity (FVC) measurements was recorded as well as FEV1, FEV1/FVC ratio, and MEF25. 
Transfer factor of the lung for carbon monoxide (TL,CO) was determined using the single-breath method (Transferscreen-II; Jaeger, Wuerzburg, Germany) and corrected for haemoglobin content. Since the correction of TL,CO for alveolar volume did not influence the results of our analysis, only uncorrected TL,CO values are reported. Measurements were performed according to the ECSC [14] and ATS [16] guidelines.

Reference values were those from a recent crosssectional study on lung function in healthy schoolchildren, aged 4-19 yrs [17, 18]. Data are expressed as an SD score ((actual result - predicted result)/population SD) and defined as pathological when $<-1.64$, corresponding to the fifth percentile [14]. Taking into account the pubertal stage of each subject at each respiratory function assessment, the SD score was corrected according to the tables reported by RosENTHAL and co-workers [17, 18]. Pubertal stage was evaluated according to the method of TANNER [19].

Three patterns of respiratory function abnormalities were defined as follows: 1) restrictive: FVC SD score $<-1.64$ with $\mathrm{FEV} 1 / \mathrm{FVC}$ index $>-1.64$; 2) obstructive: FEV1 SD score <-1.64 with FEV1/FVC index <-1.64; and 3) isolated diffusing impairment: TL,CO SD score $<-1.64$ with the other parameters in the normal range.

\section{Statistical analysis}

Repeated measures analysis of variance (ANOVA) was used to analyse changes in pulmonary function tests over time. Thirty nine FVC values were available at 3 months after BMT, 37 at 6 months, 31 at 12 months and 27 at 18 months; two different ANOVA analyses were thus performed: 1) comparing data of all 39 subjects on pre-BMT and 3 months after BMT; and 2) comparing data obtained at baseline, 3, 6, 12 and 18 months after BMT of the 27 subjects who completed the follow-up. TL,CO values were analysed in the same way, 27 patients being precluded in the first analysis, and 17 in the second; the missing $T \mathrm{~L}, \mathrm{CO}$ data with respect to FVC were due to the greater difficulty and co-operation required to perform this test than for FVC manoeuvres. Tukey's honest statistical difference (HSD) test for unequal sample size (Spjotvoll and Stoline test) and Scheffe's test were used to compare differences between groups and within groups, respectively.

ANOVA analyses were performed to evaluate the role of the type of disease and chemotherapy on lung dysfunction prior to transplant.

Logistic regression analysis (EGRET, Statistics and Epidemiology Research Co., Seattle, WA, USA) [20] was used to examine the relationship between each potentially prognostic variable and changes in lung function tests after BMT. The independent variables were: baseline respiratory function; seropositivity for HCMV; type of transplantation; different preparative regimen; development of respiratory infections; and development of acute or chronic GVHD in the first 3 months. The dependent variable was the 3 month respiratory function categorized into normal or abnormal (grouping all the above-mentioned abnormal patterns). A p-value of less than 0.05 was considered significant.

\section{Results}

The respiratory symptoms most frequently reported were cough and phlegm; they were present in 15-25\% of children at all visits. Dyspnoea was present in $<10 \%$ of cases at the 3 and 6 month visits only. Acute bronchitis was diagnosed and treated in the first 3 months in $7.9 \%$ of patients, during $3-6$ months in $8.6 \%$ of patients, 6-12 months in $10.8 \%$ and $12-18$ months in $16.1 \%$ of patients. Pneumonia was diagnosed and treated during the first 3 months of follow-up in $10.5 \%$ of patients, and 3-6 months in $8.6 \%$ of patients. One patient had pneumonia after the 6 month control and died rapidly of respiratory failure before a microbiological diagnosis was obtained and specific treatment was given. Overall, during the whole follow-up, 17 subjects never developed acute bronchitis or pneumonia, 12 had only one episode of acute bronchitis, one developed two episodes of acute bronchitis, three had one episode of acute bronchitis and one of pneumonia, and six had pneumonia. In two cases of pneumonia, sputum cultures showed Streptococcus pneumoniae or Haemophilus influenzae. All but one of these complications resolved easily after treatment. One patient developed pulmonary aspergillosis after the 12 month control and died of respiratory failure. No patients had obstructive lung disease during the follow-up.

Baseline mean values of FVC and TL,CO SD scores were in the normal range, although in the area of negative values (SD score 0- -1.64). A high percentage of patients had negative baseline values and a significant percentage already had clear pathological baseline values (SD score <-1.64), particularly for TL,CO (table 2). When the abnormal lung function parameters of each patient were grouped into patterns, only $65 \%$ of patients were normal at baseline. These subjects received chemotherapy before transplantation for a shorter time (22.8 \pm 20.3 months) than subjects with abnormal baseline lung function $(33.4 \pm 41.3$ months $)$; this difference, however, was not statistically significant. Baseline mean values of FVC and TL,CO SD score were lower, but not significantly in patients with ALL $(-0.37 \pm 1.33$ and $-1.06 \pm$ 1.43 , respectively) than in patients with AML $(0.14 \pm$ 0.57 and $-0.83 \pm 2.37$, respectively). A statistically significant decline in the mean values of FVC, FEV1 and TL,CO but not in FEV1/FVC or MEF25, was observed between baseline and 3 months after BMT. At 3 months after BMT, the percentage of subjects with clearly pathological values of FVC and TL,CO increased greatly; more than $33 \%$ of subjects had pathological values of FVC and more than $66 \%$ of TL,CO (table 2).

The 27 patients who completed the 18 month followup, showed a similar decrease of FVC after 3 months. Subsequently, the trend inverted, with recovery up to 12 months and stabilization by 18 months. ANOVA showed a statistically significant difference between times $(\mathrm{p}<0.01)$. Post hoc comparison showed that the statistical significance of the model was due to the difference between baseline and 3 month values $(\mathrm{p}<0.05)$ and to that between baseline and 6 months $(\mathrm{p}<0.05)$. Nonetheless, the mean FVC value at 18 months was still lower than at baseline (fig. 1). At 3 months, the trend of TL,CO showed a greater decrease than FVC. Despite the modest recovery at the end of the first year of follow-up, at 18 months the mean TL,CO value was still 
Table 2. - Respiratory function data at baseline and 3 months after bone marrow transplantation (BMT)

\begin{tabular}{|c|c|c|c|c|}
\hline & & SD score & $\begin{array}{c}\text { Patients } \\
\text { with values } \\
0--1.64 \\
\%\end{array}$ & $\begin{array}{c}\text { Patients } \\
\text { with values } \\
\leq 1.64 \\
\%\end{array}$ \\
\hline \multirow[t]{2}{*}{ FVC } & Baseline & $-0.22 \pm 1.12$ & 43.6 & 10.2 \\
\hline & 3 months & $-1.05 \pm 1.64 * *$ & 43.5 & 38.5 \\
\hline \multirow[t]{2}{*}{ FEV1 } & Baseline & $0.13 \pm 0.76$ & 42.0 & 7.7 \\
\hline & 3 months & $-0.76 \pm 0.96 * *$ & 43.6 & 30.7 \\
\hline \multirow[t]{2}{*}{$\mathrm{FEV} 1 / \mathrm{FVC}$} & Baseline & $0.15 \pm 0.96$ & 35.9 & 5.1 \\
\hline & 3 months & $-0.15 \pm 1.35$ & 28.2 & 15.4 \\
\hline \multirow[t]{2}{*}{ MEF25 } & Baseline & $0.24 \pm 1.80$ & 27.8 & 10.8 \\
\hline & 3 months & $-0.21 \pm 1.72$ & 42.4 & 16.2 \\
\hline \multirow{2}{*}{$T \mathrm{~L}, \mathrm{CO}$} & Baseline & $-1.21 \pm 1.73$ & 29.6 & 48.1 \\
\hline & 3 months & $-2.24 \pm 1.31 * *$ & 29.7 & 66.6 \\
\hline
\end{tabular}

Values are presented as the SD score ((actual result - predicted result)/population SD), expressed as mean \pm SD, or as percentages of patients with negative SD scores $(0--1.64)$ and pathological SD scores $(<-1.64)$. For forced vital capacity (FVC), forced expiratory volume in one second (FEV1) and FEV1/FVC, $\mathrm{n}=39$; for maximal expiratory flow at 25\% FVC (MEF25), $\mathrm{n}=37$; for transfer factor of the lung for carbon monoxide $(T \mathrm{~L}, \mathrm{CO}), \mathrm{n}=27 . * *: \mathrm{p}<0.01$ versus baseline.

clearly pathological. ANOVA showed a difference between times that was at the limit of the statistical significance $(\mathrm{p}=0.05)$. In the post hoc comparison the only statistically significant difference was between the baseline value and that at 3 months $(\mathrm{p}<0.05)$. Six patients with the worst decline in FVC were not able to carry out single-breath manoeuvres and, thus, TL,CO mean values were not processed (fig. 2). As shown by the wide sDs of the means (figs. 1 and 2), the trends in FVC and TL,CO varied greatly between subjects during the follow-up period. When abnormalities of the functional parameters of each patient during the follow-up were grouped into patterns, restrictive defects and isolated TL,CO impairment were the most prevalent. At the end of follow-up, only $42.3 \%$ of patients had normal lung function (table 3).

Of the variables that might be prognostic of the postBMT outcome, only seropositivity for HCMV was significant by the logistic regression analysis (odds ratio (OR) 10.50 ; $95 \%$ confidence interval $(95 \% \mathrm{CI}) 1.21-$

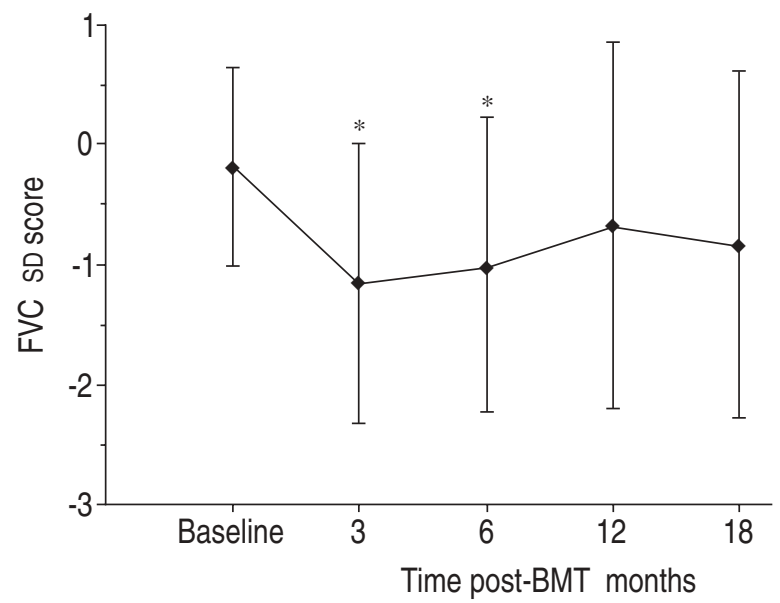

Fig. 1. - Trend of forced vital capacity (FVC) expressed as the mean \pm SD of the SD score in the group of 27 patients who completed the follow-up (baseline, 3, 6, 12 and 18 months after bone marrow transplantation $(\mathrm{BMT}))$. Analysis of variance over time: $\mathrm{p}<0.01$. *: $\mathrm{p}<0.05$, versus baseline (post hoc comparison).

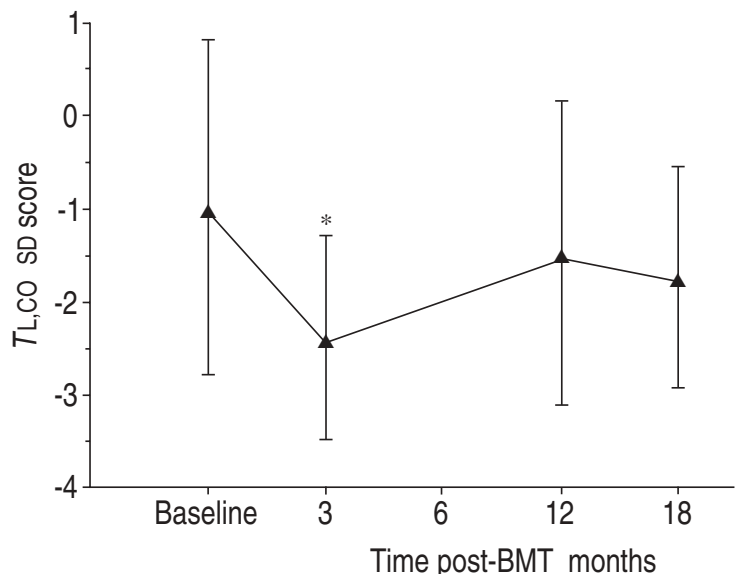

Fig. 2. - Trend of transfer factor of the lung for carbon monoxide $(T \mathrm{~L}, \mathrm{CO})$ expressed as the mean \pm SD of the SD score in the group of 17 patients who completed the follow-up with all measurements (baseline, 3, 6, 12 and 18 months after bone marrow transplantation (BMT)). Analysis of variance over time: $\mathrm{p}=0.05$. *: $\mathrm{p}<0.05$, versus baseline (post hoc comparison).

91.03; $\mathrm{p}<0.05)$. The presence of chronic GVHD also played a role, although it was not significant (OR 3.42, 95\% CI 0.28-40.95; $\mathrm{p}=\mathrm{NS}$ ). Baseline lung function $\mathrm{ab}-$ normalities, type of transplant (allogeneic versus autologous), type of conditioning regimen (with TBI versus no TBI) and development of respiratory infections in the first 3 months did not significantly influence the respiratory function at 3 months post-BMT.

Figure $3 \mathrm{a}$ shows that the individual FVC values of the subjects who received autologous BMT are in the normal range during the follow-up period in all but one patient (who had an episode of acute bronchitis before the 12 month examination). In the group of subjects given an allogeneic BMT without acute or chronic GVHD (fig. 3b) the trend in the FVC values was similar in all patients, all being in the negative range and many in the pathological range even at 12 and 18 months. In the group of subjects with acute GVHD who did not subsequently develop chronic GVHD (fig. 3c) there was a decrease of FVC at 3 months in $~ 50 \%$ of subjects and a progressive recovery in all of but one patient; all these patients were treated with high doses of steroids. The subject with the worst trend had an HCMV infection reactivation 5 months after BMT; at the end of follow-up an improvement was observed, but the values were still in the pathological range. In the group of subjects with acute and chronic GVHD (fig. $3 d$ ), at 3 and 6 months, FVC values were even lower than in the group with acute GVHD only; the recovery at the end of follow-up varied greatly in the different patients.

Table 3. - Patterns of respiratory function abnormalities during the follow-up

\begin{tabular}{lccccc}
\hline & \multicolumn{5}{c}{ Patients \% } \\
\cline { 2 - 6 } Pattern & Baseline & 3 & 6 & 12 & 18 \\
& & months & months & months & months \\
\hline Normal & 70.4 & 25.9 & 25.9 & 38.0 & 42.3 \\
Restrictive & 3.7 & 37.0 & 25.9 & 14.8 & 30.8 \\
Obstructive & 0 & 7.4 & 14.8 & 18.5 & 7.7 \\
Isolated TL,CO & 23.5 & 41.2 & 41.2 & 29.4 & 31.2 \\
\hline
\end{tabular}

+: percentages of patients with transfer factor of the lung for carbon monoxide $(T \mathrm{~L}, \mathrm{CO})$ measurements during the complete follow-up. 

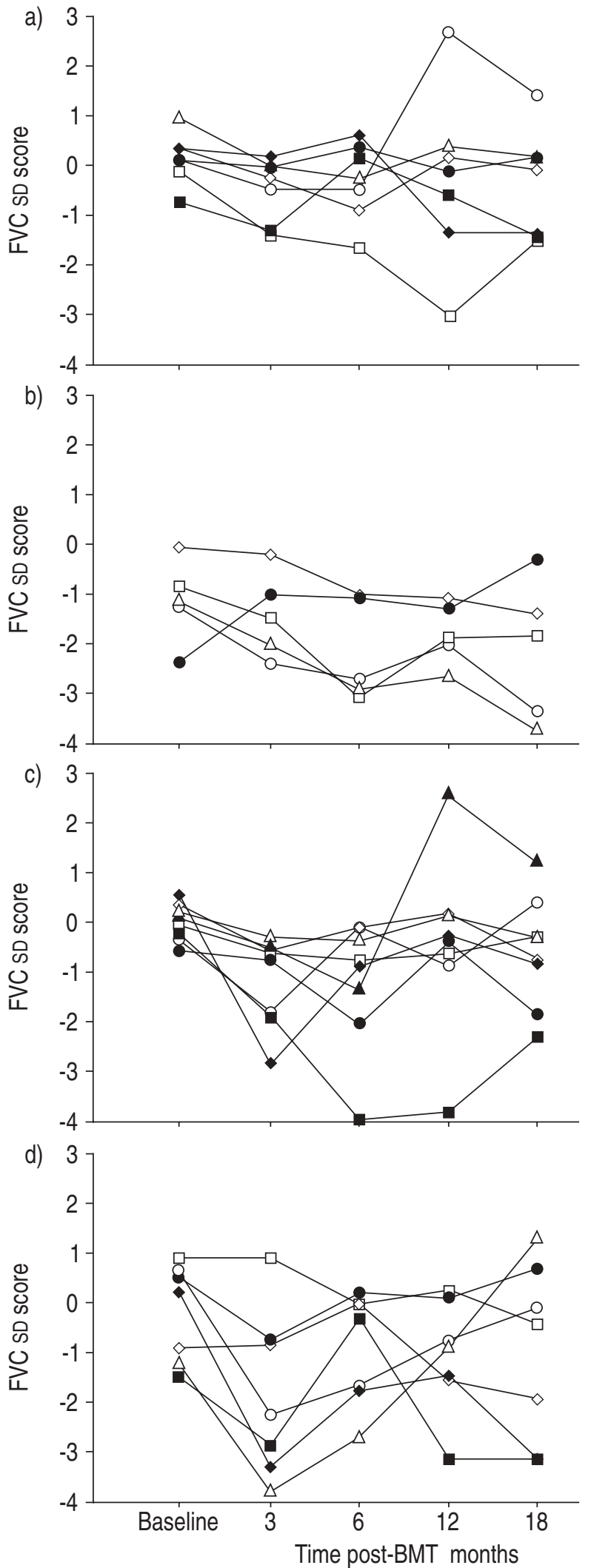

Fig. 3. - Individual plots of forced vital capacity (FVC) expressed as SD score values at baseline, 3, 6, 12, and 18 months after bone marrow transplantation (BMT) of the 27 subjects who completed the follow-up, subdivided into: a) seven patients given an autologous BMT; b) five patients given an allogeneic BMT with no acute or chronic graft-versus-host disease (GVHD); c) eight patients with acute GVHD; and d) seven patients with acute and chronic GVHD.

\section{Discussion}

This study expands current knowledge of the respiratory consequences of BMT in children. Acute bronchitis and pneumonia were found to be frequent, but easily resolved after treatment in all but one case. An important finding of our study is the absence of obstructive lung disease in the period of our follow-up, at least as defined by the literature [4]. There is previous evidence that children may fare better than adults in this respect, but the data are still conflicting $[4,8]$. We found that children with acute and particularly chronic GVHD had more lung function abnormalities than the other patients but no clinically evident respiratory manifestations. Because lung function abnormalities were at least in part reversible, either spontaneously or after high doses of prednisone, we think that obstructive lung disease can be an easily controlled problem by early treatment of acute and chronic GVHD and accurate and frequent monitoring of respiratory symptoms and lung function.

One of the most important findings of this study is that there is variability of outcomes in terms of respiratory function: few patients had no or minimal abnormalities while many others had more permanent injury as they developed important restrictive defects with significant impairment of diffusion capacity. At 18 months, progressive recovery of lung function was observed, although only $\sim 40 \%$ of the patients reached values within the normal range.

We found that a moderate percentage of our patients already had lung function abnormalities at baseline, which may have resulted from previous treatments. In fact, baseline lung function was more impaired in patients with ALL, who had been treated for a longer time with chemotherapy before having their transplant, than in those patients with AML. Unfortunately, we could not find any statistically significant association, probably due to the different histories and to the wide range in duration of treatments before admission to our clinic.

At present, the exact effects of factors potentially influencing lung function after BMT (i.e. baseline lung function abnormalities, cytoreductive regimens, immunosuppression, infections, type of transplant and GVHD) have not been completely clarified; the only variable that we found to be prognostic was seropositivity to HCMV, as already observed by QuigLey et al. [5]. The power of the statistical analysis of our sample, as for all the other studies in the literature, is limited by the relatively small sample size, due to the difficulty in recruiting a sufficiently large population of such children in a short period of time. As the different potential risk factors are not distributed homogeneously, the detection of differences between groups and significant interactions becomes difficult. We think that this limitation had a particular influence on the evaluation of the effect of type of transplant (29 patients with allogeneic, 10 with autologous BMT). Indeed, when we evaluated the individual plots of FVC against time for the different groups of subjects, as suggested by MATTHEws et al. [21], we found that the group of subjects who received autologous transplant showed a trend that was constantly within the normal range (fig. 3a), while the group of subjects who received allogeneic transplants (fig. 3b) had worse values of FVC independent of acute 
and chronic GVHD occurrence. Immune-mediated damage to the recipient lung might explain the more marked impairment in lung function in allogeneic BMT patients. Drug regimen toxicity probably plays an important role; we were unable to confirm this, because the group of children who received BMT for nonmalignant diseases was too small to allow any comparison and was, thus, not included in this study. In any case, because of the different individual responses, the amount of lung damage due to drugs cannot be predicted. No statistically significant difference was found between patients who underwent TBI and those who did not, in the conditioning regimen. Although high-dose radiation is a known cause of lung function impairment, we think, as do Quigley et al. [5], that irradiation over several days in fractionated doses can result in better preservation of lung function. In our statistical model, the presence of respiratory infections in the first 3 months was not found to be prognostic; thus, the prolonged lung function abnormalities could not be the result of recurrent bronchitis or pneumonia. Unexpectedly, prior lung damage was not found to be significant in predisposing to more severe transplant injury. A previous study in adults found a significant correlation between pulmonary function tests before BMT and risk of death [22], while another study only found a significant correlation with a higher risk of pulmonary complications [23]. The important consequence of this unexpected result is that it may be possible to include subjects with even severe lung function abnormalities in a transplantation programme.

In conclusion, respiratory infections were frequent complications after bone marrow transplantation, but resolved quickly in almost all cases. Obstructive lung disease might be controlled by early diagnosis and treatment. In spite of this, after bone marrow transplantation, a significant proportion of children develop restrictive defects and impairment of diffusion capacity that are still present at the end of follow-up in more than 50\% of cases. These abnormalities are only partially predicted by the seropositivity to human cytomegalovirus and by the development of graft-versus-host disease. Owing to the lack of precise information on other predictive factors, we recommend careful monitoring in all subjects in this period, even in the absence of respiratory symptoms. As the lung function in many patients is still in the pathological range at 18 months, longerterm follow-up studies are needed to evaluate the final outcome in these children [7, 24].

\section{References}

1. Clark JCL, Harness J, Hertz MI. Idiopathic pneumonia syndrome after bone marrow transplantation. Am Rev Respir Dis 1993; 147: 1601-1606.

2. Breuer R, Iossos IS, Berkman N, Or R. Pulmonary complications of bone marrow transplantation. Respir Med 1993; 87: 571-579.

3. Stokes DC. Pulmonary complications of tissue transplantation in children. Curr Opin Pediatr 1994; 6: 272-279.

4. Schultz KR, Green GJ, Wensley D, et al. Obstructive lung disease in children after allogeneic bone marrow transplantation. Blood 1994; 84(9): 3212-3220.

5. Quigley PM, Yeager AM, Loughlin GM. The effects of bone marrow transplantation on pulmonary function in children. Pediatr Pulmonol 1994; 18: 361-367.
6. Kaplan EB, Wodell RA, Wilmott RW, Leifer B, Lesser ML, August CS. Late effects of bone marrow transplantation on pulmonary function in children. Bone Marrow Transplantation 1994; 14: 613-621.

7. Arvidson J, Bratteby LE, Carlson K, et al. Pulmonary function after autologous bone marrow transplantation in children. Bone Marrow Transplant 1994; 14: $117-$ 123.

8. Kaplan EB, Wodell RA, Wilmott RW, Leifer B, Lesser ML, August CS. Chronic graft-versus-host disease and pulmonary function. Pediatric Pulmonology 1992; 14: 141-148.

9. Paolucci G, Masera G, Vecchi V, et al. Treating childhood acute lymphoblastic leukemia (ALL): summary of ten years' experience in Italy. Med Pediatr Oncol 1989; 17: 83-91.

10. Amadori S, Ceci A, Comelli A, et al. Treatment of acute myelogenous leukemia in children: results of the Italian co-operative study AIEOP/LAM 8204. J Clin Oncol 1987; 5: 1356-1363.

11. Storb R, Deeg HJ, Whitehead J, et al. Methotrexate and cyclosporine compared with cyclosporine alone for prophylaxis of acute graft-versus-host disease after marrow transplantation for leukemia. $N$ Engl J Med 1986; 314: 729-735.

12. Glucksberg H, Storb R, Fefer A, et al. Clinical manifestations of graft-versus-host disease in human recipients of marrow from HLA-matched sibling donors. Transplantation 1974; 18: 295-304.

13. Shulman HM, Sullivan KM, Weiden PL, et al. Chronic graft-versus-host syndrome in man. Am J Med 1980; 69: 204-217.

14. Quanjer PhH, Tammeling GJ, Cotes JE, Pedersen OF, Peslin R, Yernault J-C. Report Working Party. Standardization of lung function tests. Eur Respir J 1993; 6: Suppl. 16, 5-40.

15. American Thoracic Society. Standardization of spirometry. Am J Respir Crit Care Med 1995; 152: 1107-1136.

16. American Thoracic Society. Single-breath carbon monoxide diffusing capacity (transfer factor). Am J Respir Crit Care Med 1995; 152: 2185-2198.

17. Rosenthal M, Bain SH, Cramer D, et al. Lung function in white children aged 4 to 19 years: I. Spirometry. Thorax 1993; 48: 794-802.

18. Rosenthal M, Cramer D, Bain SH, Denison D, Bush A, Warner JO. Lung function in white children aged 4 to 19 years: II. Single breath analysis and plethysmography. Thorax 1993; 48: 803-808.

19. Tanner JM. Growth at Adolescence. 2nd Edn. Oxford, Blackwell, 1962.

20. Egret (reference manual). Statistics and Epidemiology Research Corporation, Seattle, Washington, USA 1993.

21. Matthews JNS, Altman DG, Campbell MJ, Royston P. Analysis of serial measurements in medical research. BMJ 1990; 300: 230-235.

22. Crawford SW, Fisher L. Predictive value of pulmonary function tests before marrow transplantation. Chest 1992; 101: $1257-1264$

23. Ghali R, Szidon JP, Thompson L, Nawas YN, Dolce A, Kaizer H. Evaluation of pulmonary complications after bone marrow transplantation: the role of pretransplant pulmonary function tests. Bone Marrow Transplant 1992; 10: 359-365.

24. Nenadov Beck M, Meresse V, Hartmann O, Gaultier C. Long-term pulmonary sequelae after autologous bone marrow transplantation in children without total body irradiation. Bone Marrow Transplant 1995; 16 : $771-775$. 